\title{
Two operations In character recognition: A partial replication
}

\author{
GERALD W. BRACEY 1 \\ EDUCATIONAL TESTING SER VICE
}

\begin{abstract}
Subjects carried out a choice reaction time experiment in which they were required to press one lever if a tachistoscopically flashed numeral was a member of a previously memorized list of numerals and a second lever if it was not a member of the list. The number of numerals in the list was varied from one to four. On half of the trials the numeral was partially masked by a pattern of visual noise. For one group a single fixed pattern of noise was used; for a second group four patterns presented in random order were used. Reaction time from the onset of the mumeral to the lever press was found to be a linear function of list size, confirming Stermberg's hypothesis that the list is scanned serially to determine a match. Partial masking was found to add a constant increment to reaction time for both groups, independent of set size. The increment was equal for both groups. The constancy of the reaction time increase contradicts Sternberg's results in which the increment was found to be, in part, a function of list size. The results of the present study indicate that one cannot dismiss the possibility that Ss identify the character to the point of labeling prior to an attempt to determine list membership.
\end{abstract}

In a recent paper, Sternberg (1967) describes two operations that are carried out whenever a $S$ attempts to determine whether a given stimulus (such as a flashed numeral) is or is not a member of a previously memorized list of such stimuli. The proposed operations are: (1) a serial and exhaustive scan through the memorized list during which the input stimulus is compared to the list members to determine the presence or absence of a match, and (2) a preprocessing operation that refines the raw image to some such form as a normalized template, a set of distinctive features, or an acoustic representation (label).

Evidence for the two operations comes from choice reaction time experiments. Briefly, the $S$ memorizes a list of numerals, views one of the numerals, 0 through 9 , and makes one response if the presented numeral is a member of the list and a second response if it is not. Reaction time is found to be a linear function of the size of the memorized list, indicating a serial scan of memory. Moreover, the curves, plotted separately for "target" stimuli (a target stimulus is a member of the list) and nontarget stimuli, have equal slopes, indicating that the scan is exhaustive (see Stemberg, 1966, 1967, for details of this argument).

On half of the trials, the stimulus is not presented alone, but is partially masked by a noise pattern. Sternberg describes two ways in which a $S$, under these conditions, might attempt to recognize and categorize the presented numeral: (1) He might attempt the scan without the preprocessing operation, with the entire stimulus complex including the masking noise. If it is assumed that each comparison in the scan is slowed down by some factor, $\Delta$, then the distance between the curve for intact stimuli and that for masked stimuli will be a function solely of the size of $\Delta$ and the length of the list; the longer the memorized list, the greater the distance between the two curves. This is shown as Model I in Fig. 1. The intercepts of the two curves, taken as a hypothetical point where no comparisons are made, are identical, but the slopes diverge as a function of the magnitude of $\Delta$. (2) He might perform some preprocessing operations to refine the stimulus
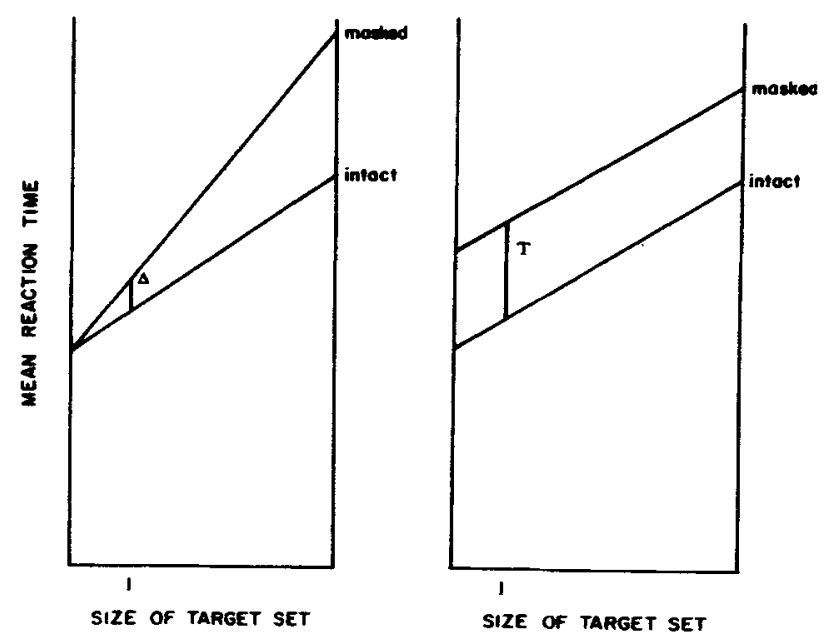

Fig. 1. Two idealized models for the effects of noise on reaction time in a choice resction-time situation (modified from Sternberg, 1967).

before initiating the search. This process is assumed to take some time, $T$, but is carried out only once for any given input and, hence, adds a constant to the reaction time. If such is the case, the relationship between the two curves will resemble Model II of Fig. 1 .

Stemberg's (1967) results indicated that the data fit neither of the idealized models perfectly. He found not only a large intercept effect, consistent with Model II, but also a significant, although small, slope effect, consistent with Model I. However, when the same Ss repeated the experiment 1 week later, the slope effect disappeared. These results are somewhat difficult to interpret, but one possible explanation is that the filtering operation develops over the course of the experiment and becomes highly efficient with practice. This interpretation seems more reasonable in view of the fact that Stemberg's masking noise was a single pattern superimposed on the numeral. If this interpretation is correct, one might expect that a masking pattern that varied unpredictably from trial to trial would produce a greater slope effect than a single pattern. The following experiment tests this possibility.

\section{Subjects}

Twenty-four male undergraduates enrolled in the introductory psychology course at Stanford University served as Ss. Ss were not paid for participating in the experiment per se, but were informed that a $\$ 5.00$ bonus would be awarded to the person having the fastest mean response time. They were also informed that $0.1 \mathrm{sec}$ would be added to their mean response time for each error. Ss were divided into two groups of 12 . Group $F$ received a single fixed pattern of noise on trials when the stimulus was masked. Group V received, randomly, one of four patterns. 


\section{Stimulus Materials}

The stimuli consisted of the numerals 0 through 9 mounted onto a strip of heavy, hard-finish white paper from a Letraset 258 lettering set. The numerals were 48-point type, approximately $5 / 8$ in. high. The noise patterns were created by mounting dots from a Transartype lettering set, T3040, onto a sheet of clear acetate that was placed in front of the numerals when desired. (These materials differ somewhat from those used by Sternberg, 1967 ; in his experiment numerals were presented by a Burroughs Nixie tube and the masking was produced by a split beam of light.) For Group F, a single pattern of noise was constructed by dividing a 1-in. square into an 8 by 8 matrix and filling alternate squares in checkerboard fashion. Only the dots appeared as noise; the outline of the matrix was used only to position the dots appropriately. For Group V, the variable noise group, four noise patterns were constructed. The 8 by 8 matrix for Group $V$ was filled by randomly drawing 32 of the numbers 1 through 64 and filling the appropriate squares. Two constraints were placed on the matrices: (1) There could be no more than five nor fewer than three dots in any row or column. (2) There could be neither four dots nor four spaces adjacent to each other in such a manner as to, themselves, form a square. These constraints prevented the complete occlusion or complete disclosure of the numeral.

\section{Apparatus}

Stimuli were presented by a Gerbrands two-field tachistoscope, Model Dt-Sp. Field 1 was used as a fixation field; it was on continuously except during presentation of the stimuli and was black except for a pinpoint of light that corresponded to the center of the display field. The onset of Field 2 displayed the stimulus in the center of the field and simultaneously started a standard timer, which was stopped when $S$ depressed one of two levers attached to a response box. Ss depressed the levers with a downward stroke of the thumb.

\section{Procedure}

The stimuli were presented in one block of 60 trials, followed by three blocks of 90 trials each. For the first block, a practice block, the size of the memorized list (hereafter referred to as the target set or "s") was always three and consisted of the numerals 1,6 , and 9 . The remaining three blocks were some permutation of the possible orders of $s=1,2$, or 4 . Each block was divided into two equal subblocks, one subblock consisting of intact stimuli, the other of stimuli masked by noise. Half of the Ss received a subblock of masked stimuli first, half received intact stimuli first. Ss were given approximately $2 \mathrm{~min}$ rest between each subblock. A trial lasted approximately $6 \mathrm{sec}$. It consisted of a warning period of $2 \mathrm{sec}$, presentation of the stimulus for $100 \mathrm{msec}$, the S's response, feedback to the $S$ as to whether he was right or wrong (but not what his time had been), and repositioning of the stimuli. The warning signal was the sounds made by the tachistoscope prior to presentation of the stimulus. Ss were instructed in the use of these sounds as a preparatory signal and were given several trials prior to the start of the experiment in which to practice coordinating the sounds with the appearance of the numerals.

Response uncertainty was constant across all subblocks of trials. For each subblock, members of the target set appeared 12 times. This meant that specific numerals had to occur with different frequencies depending on the size of the target set of which they were members: There were 12 of whatever numeral was being used as $s=1,6$ each of whatever was being used as $s=2$, and 3 each of whatever was being used as $s=4$. Stimulus uncertainty was held constant across all subblocks of trials by having the specific numerals appear with the same frequency in all subblocks whether or not they belonged to the target set for a particular block. For the subblocks of $s=3$, which were shorter than the other subblocks, the number of target items was reduced proportionately.

To control for any peculiarities of individual numerals, a permutation of the seven numerals being used in the experimental blocks was taken, which was then shifted "one-to-the-right" for each S. Thus, for example, if the permutation were 5384072 , the first $S$ would get 5 for $s=1 ; 3$ and 8 for $s=2$; and $4,0,7$, and 2 for $s=4$. $S 2$ would get 2 for $s=1 ; 5$ and 3 for $s=2$; and $8,4,0$, and 7 for $s=4$. A permutation was chosen so that for $s=2$ the members were never in adjacent serial order (e.g., 3 and 4), and that for $s=4$ no obvious coding scheme (e.g., "first four even numbers") was available. Prior to the start of each subblock, the $E$ read the members of the target set in ascending serial order and repeated them if the $S$ so requested or if he appeared to be confused.

With the above scheme, the usage of particular numerals can be balanced across all values of $s$ in multiples of seven Ss. However, it was felt that the order effects of set size presentation and intact first vs masked first were likely to be of far greater importance in this experiment than peculiarities of specific numerals. These effects can be balanced in multiples of $12 \mathrm{Ss}$. Rather than carry out the experiment on $84 \mathrm{Ss}$ to balance both effects, $12 \mathrm{Ss}$ were run, compromising the frequency of the appearance of numerals in the various set sizes. With the specific permutation used, the numerals 0 and 3 appeared only once for $s=1$, while the remaining numerals appeared twice; for $s=2$, the numerals 0,3 , and 7 appeared four times, while the numerals $2,4,5$, and 8 were used three times each; for $s=4$, the numeral 5 appeared six times, while the remaining numerals were each used in seven target sets.

The order of presentation of the numerals was randomized for each subblock; each $S$ had a different randomization. Ss were assigned randomly to the 12 possible set size orders and stimulus conditions.

\section{Treatment of the Data}

For the purposes of analysis, the first two subblocks of trials (with $s=3$ ) were discarded; also discarded were the first 10 trials of all remaining subblocks, $\mathrm{S}$ errors, $\mathrm{E}$ errors, and apparatus failures. There remained, after such removals, approximately 31 reaction times per subblock per $S$.

For each $S$, means were calculated for each subblock. For each group, regression lines were fitted to these means, separate lines being fitted for intact and masked stimuli. To determine whether or not the addition of noise altered the slope or the intercept of the regression line for intact stimuli, the following procedure was used. For each group a mean reaction time was calculated for the entire experiment. The means for the separate blocks were then subtracted from the grand mean and the resulting numbers taken as measures of practice independent of set-size order or stimulus-condition order (since across the experiment these orders are balanced within each block). Following this, the means of a pair of Ss who had had the same order of set-size presentation, but a different order of intact and degraded presentation, were averaged and corrected for the practice effects of each block. A regression line was then fitted through these corrected values. This procedure yielded, for each group of $12 \mathrm{Ss}$, six regression lines for intact stimuli and six regression lines for degraded stimuli, one each for each pair of Ss. For each group as a whole, differences between the slopes and intercepts of the lines for intact and degraded stimuli were then tested, within each group, with a t test for correlated means; between groups, with a $t$ test for independent means.

\section{Results}

Figure 2 shows the mean reaction time for each set size and 


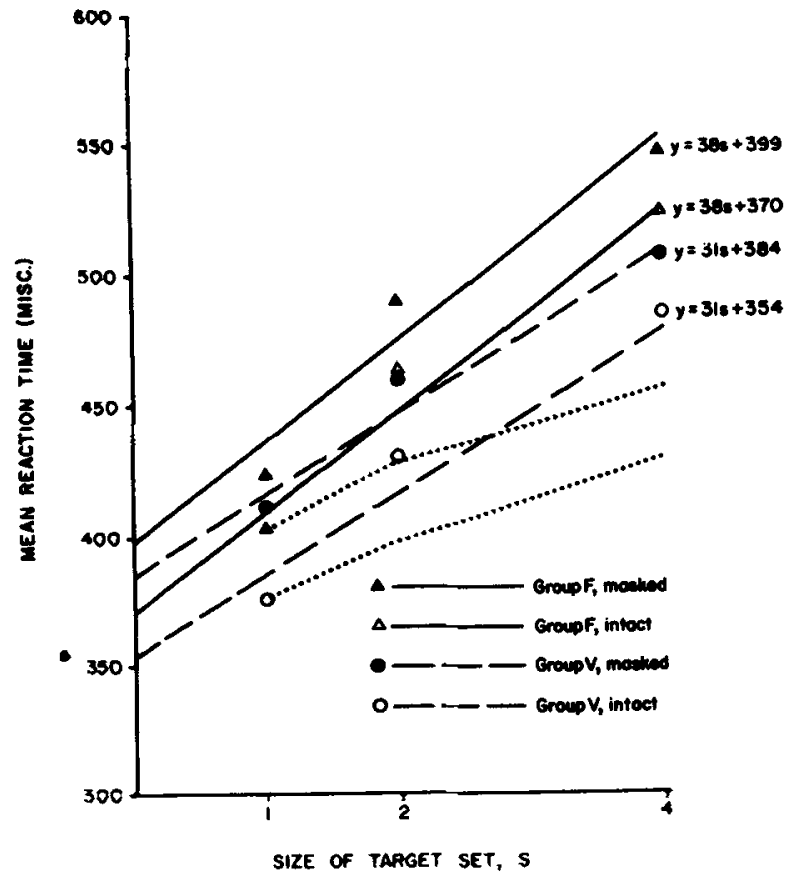

Fig. 2, Reaction time as a function of size of target set and stimulus condition. Regression lines have been plotted through the means and their equations shown. Also shown are the upper bounds for parallel processes for Group $F$ (upper dotted curve) and Group V (lower dotted curve).

stimulus condition for the two groups as well as the regression lines through the means and their corresponding equations. For both groups, the masking of numerals by the superimposition of a pattern of visual noise produces a significant increase in the intercept of the regression lines $[t(5)=3.19$ and $7.69 ; p<.025$ and .001 , one-tailed; for Groups F and V, respectively]. Within each group, the slopes of the intact and masked stimuli are identical, $38 \mathrm{msec} / \mathrm{unit}$ of $\mathrm{s}$ and $31 \mathrm{msec} / \mathrm{unit}$ of $\mathrm{s}$ for Groups $\mathrm{F}$ and $\mathrm{V}$, respectively. Across groups, the values of the slopes for intact stimuli do not differ significantly $[t(22)=2.04, p<.10]$. Since the values of the slopes for the two groups do not change in the presence of noise, a t statistic testing the difference between differences yields a value of 0.0 . The values of the intercepts for intact stimuli do not differ for the two groups $(p>.05)$. The effect of noise on these intercepts is nearly identical for the two groups ( 29 and $30 \mathrm{msec}$ ); hence, the resulting $t$ statistic for the difference between differences is again very small.

Because of the consistency with which the means appear to depart from linearity, a test for such departure was made. None of the resulting $F$ s are significant $[F(1,33)=3.41$ and 2.05 for Group $F$, intact and masked stimuli, respectively; $F(1,33)=1.35$ and 1.62 for Group V, intact and masked stimuli, respectively]. Error rates are shown in Table 1.

\section{Discussion}

The slopes of the regression lines are remarkably close to those reported by Sternberg $(1966,1967)$, as are the intercepts for intact stimuli. The increase in reaction time with increasing set size rules out the possibility that comparisons are made by parallel processes that start and finish simultaneously. ${ }^{2}$ As Sternberg (1966) has pointed out, however, reaction time could increase if the comparison times were independent, not equal, and the response was initiated only after the termination of the
Table 1

Error Rates as a Function of Set Size and Stimulus Condition

\begin{tabular}{|c|c|c|c|c|c|c|}
\hline & \multicolumn{6}{|c|}{ Per Cent Error } \\
\hline & \multirow{2}{*}{\multicolumn{3}{|c|}{$\begin{array}{l}\text { Group F } \\
\text { Set Size }\end{array}$}} & \multirow{2}{*}{\multicolumn{3}{|c|}{$\begin{array}{l}\text { Group V } \\
\text { Set Size }\end{array}$}} \\
\hline & & & & & & \\
\hline & 1 & 2 & 4 & 1 & 2 & 4 \\
\hline Intact & 1.2 & 1.2 & 2.4 & 1.1 & 3.2 & 3.2 \\
\hline Masked & 2.4 & 1.2 & 1.9 & 2.3 & 1.3 & 2.6 \\
\hline
\end{tabular}

slowest. By applying the equations of Hartley and David (1964) to his data, Sternberg (1966) was able to show that the upper bound for such parallel processes was not sufficiently high to account for the observed increases. This bound, applied to the present data, leads to the same conclusion, supporting the hypothesis that the memory scan proceeds serially. ${ }^{3}$

Although the slopes of the regression lines and the intercepts for intact stimuli are similar to those reported by Sternberg $(1966,1967)$, the increase in intercept as a result of masking the stimuli is quite different. Sternberg (1967) reports an average increase in intercept of $67 \mathrm{msec}$, whereas in the present study the increases are $30 \mathrm{msec}$. The reasons for these differences are not clear. The stimuli of both experiments were approximately the same size (.6 in.) and at the same distance from the $S$ (29 in. in Sternberg's experiment; 27 in, in the present study). Stemberg's stimuli were luminous while the numerals used in the present study were illuminated, but it is difficult to explain why this difference should affect masked, but not intact, stimuli. There are, however, other differences in the experimental situations that could be of importance. Sternberg's noise pattern was a "true" checkerboard with alternate squares filled. In the present study, the squares of the matrix were "filled" with circular dots, introducing both a qualitative and quantitative difference. Moreover, the stimuli in Sternberg's (1967) experiment were available to the $S$ for a duration of $44 \mathrm{msec}$, as compared with $100 \mathrm{msec}$ in the present study. Sternberg observed that both the slope and intercept of the function in his 1967 study are practically identical to those of an earlier study (1966) in which the presence of the stimulus was response-terminated, and he concluded that the duration of the stimulus is of little consequence in determining the values of the function. However, all of the stimuli in the 1966 study were presented intact, and it is possible that temporal factors may be more important under conditions of impoverished viewing.

The absence of any slope change under conditions of masked stimuli, and the equality of the intercept for both a fixed and variable pattern of noise, indicate that it is no easier to filter a single fixed pattern of noise than a variable pattern, the variants of which appear unpredictably. The absence of slope effect for either group indicates that the operations involved do not develop over the course of the experiment. The possibility that a filter for dots, per se, develops cannot, however, be ruled out.

The fact that for neither group did the presence of noise produce any change in the slopes of the functions contradicts Sternberg's (1967) finding and is a datum of some theoretical importance. Stemberg uses the increase in slope to argue against theories that hold that the result of any filtering process is a label or some form of imageless concept, as well as against "marker" theories of memory search. His argument is essentially intuitive: it is difficult to conceive of how noise might reside in a label or marker. The present data cannot, of course, be considered as strong evidence for label or marker theories as they can also be made consistent with a template or distinctive-features theory. For example, if one takes the magnitude of the intercept effect, which in the present study is less than half that found by 
Sternberg, as an indication of difficulty of filtering, then it is possible to argue that the absence of slope effects in the present study is the result of an easier filtering task. It is plausible that masking that produces a greater "strain" on a filter might also result in that filter's ceasing to operate with $100 \%$ efficiency in removing the noise. Nevertheless, the failure to observe any increase in slope implies that theories using labels, imageless concepts and markers, cannot at present be ruled out as possibilities.

\section{REFERENCES}

HARTLEY, H. O. \& DAVID, H. A. Universal bounds for mean range and extreme observation. Annals of Mathematical Statistics, 1964, 25, 85-99.

STERNBERG, S. High speed scanning in human memory. Science, 1966, $153,652-654$.

STERNBERG, S. Two operations in character recognition: Some evidence from reaction-time experiments. Perception \& Psychophysics, 1967, 2, 45-53.

\section{NOTES}

1. Address: Educational Testing Service, Princeton, New Jersey 08540. 2. Except in the case where increasing the number of comparisons linearly increases the time required for the completion of each comparison. Although such a possibility seems remote, theoretically it cannot be discarded.

3. The calculations of the upper bounds of parallel processes are based on the assumption that the variance of the total reaction time is not smaller than the variance of the comparison processes. To apply the Hartley-David formula, one needs to know the variance of the comparison processes. Since this quantity is not known, the variance of the total reaction time was used as a substitute in Sternberg (1966; personal communication, 1969) and in the present study.

(Accepted for publication April 16, 1969.) 